\title{
Research on mechanism of the effect of charred hawthorn on digestive by SCF /c-kit pathway
}

\author{
Qian He \\ Southwest Jiaotong University \\ Cui Liu \\ Southwest Jiaotong University \\ Ting Wang \\ Southwest Jiaotong University \\ Jingying Sun \\ Southwest Jiaotong University

\section{Xia Zhou} \\ Southwest Jiaotong University

\section{Yun Wang} \\ Southwest Jiaotong University

\section{Li Ma} \\ Oklahoma State University \\ Jun Wan ( $\square$ yanruyulab@126.com ) \\ Southwest Jiaotong University
}

\section{Research}

Keywords: Hawthorn decoction, Odor treatment, Diet-induced dyspepsia, Electroencephalography, Gastrointestinal motility , SCF/c-kit pathway

Posted Date: January 5th, 2021

DOI: https://doi.org/10.21203/rs.3.rs-138593/v1

License: (c) (i) This work is licensed under a Creative Commons Attribution 4.0 International License. Read Full License 


\section{Abstract \\ Background}

Hawthorn is the dry ripe fruit of Crataegus pinnatifida Bge in rose family which is a traditional Chinese medicine(TCM) for High-calorie-diet-induced dyspepsia (HC-DID). This study aimed to investigate whether the charred hawthorn coupled with its odor could alleviate HC-DID by brain-gut interaction and stem cell factor(SCF) /c-kit pathway.

\section{Methods}

Rats were randomly divided into 7 groups: control group, model group, cisapride group, hawthorn group (HT), charred hawthorn group (CHT), odor of charred hawthorn (OCHT), CHT + OCHT group. HC-DID rat model was established by high calorie diet for 10 days, hawthorn decoction was administered by gavage, and the self-developed solid drug odor delivery device was used for odor administration. The body weight, food intake, gastrointestinal motility, gastric fluid and gastric acid flow index were determined. HE staining was used to observe the pathological changes of rats. Electrophysiology was used to evaluate the effect of hawthorn combined with its charred odor therapy on electroencephalogram and Electrogastrogram. The SCF and c-kit levels or expressions by immunohistochemistry(IHC), enzyme linked immunosorbent assay (ELISA).

\section{Results}

We found that the odor of charred hawthorn ( $\mathrm{OCHT}$ ) affects the brain (central nervous system) of rats, and hawthorn decoction (raw hawthorn and charred hawthorn) coupled with the odor of charred hawthorn alleviated the symptoms of dietinduced dyspepsia in rats by modulating SCF/c-Kit pathway.

\section{Conclusion}

Thus, we concluded that odor of charred hawthorn has therapeutic effect on dyspepsia and hawthorn may alleviate dyspepsia related symptoms by affecting SCF/c-Kit signal pathway.

\section{Background}

Dyspepsia is usually referred to as "Shi $\mathrm{Ji}$ " in Chinese medicine, derives from in the most part the unreasonable eating behavior followed by food stagnate in the gastrointestinal tract, resulting in affecting the digestive function ${ }^{[1-3]}$. Clinical and animal studies have shown that the symptoms of "Shi Ji", that is called as HC-DID, are similar to that of functional dyspepsia (FD), especially in gastrointestinal motility, gastric acid secretion, etc. ${ }^{[4,5]}$. FD and/or "Shi Ji" (HC-DID) are common gastrointestinal diseases, gastrointestinal motility disorder is the main clinical manifestations. Slow wave is the basis of gastrointestinal motility, interstitial cells of Cajal (ICC) is the pacemaker cells of the slow wave, and also the only factor that affects slow wave ${ }^{[6,7]}$. C-Kit is a specific marker of gastrointestinal ICC, whose receptor called for stem cell factor receptor (SCF), SCF/C-Kit pathway plays an important role in maintaining the proliferation, differentiation and phenotype of ICC, which is closely linked with gastrointestinal motility ${ }^{[8,9]}$. Therefore, SCF/C-Kit pathway may be involved in the pathological process of FD or "Shi Ji" (HC-DID) through the modulation of ICC.

Hawthorn is the dry mature fruit of Crataegus pinnatifida Bge which mainly contains flavonoids and organic acids [10] Hawthorn mainly clinically used for treating "Shi Ji" (HC-DID), organic acids, amylase and protease in hawthorn, have been show to possess digestive properties ${ }^{[11]}$. Stir-frying is one of the processing methods of traditional Chinese medicine (TCM), which has influence on physicochemical properties, curative effect and function of TCM ${ }^{[12]}$. The organic acid content in 
charred hawthorn was significantly reduced in the stir-frying process, but the indigestion-action of charred hawthorn is stronger than that of raw hawthorn. Because of significant differences among odor in the stir-frying process of hawthorn, the change of odor is an important criterion for its curative effect. In our previous studies, 11 main burnt volatile constituents (odor) in charred hawthorn significantly increased compared to that of hawthorn by headspace solid-phase microextraction gas chromatography-mass spectrometry (HS-SPME-GC/MS) analysis. Furthermore, fourin 11 constituents

had been reported to have the function of digestion ${ }^{[13-14]}$ (Supplementary Materials TableS1 and Fig.S1). We therefore speculated that the odor of charred hawthorn may treat diet-induced dyspepsia by activating the central nervous system (brain).

Hawthorn can regulate gastrointestinal motility and treat "Shi Ji" (HC-DID), which is well known in China, however its mechanism has rarely been studied deeply. Research on the digestive mechanism of hawthorn have focused on the chemical composition, but not on the odor of charred hawthorn. Therefore, we firstly studied the effect of the odor of charred hawthorn on the brain regions related to digestion in rats, followed by the mechanism of hawthorn, including hawthorn decoction, charred hawthorn decoction and the odor of charred hawthorn, on digestion by regulating gastrointestinal motility and SCF/c-kit pathway.

\section{Materials And Methods}

\subsection{Hawthorn preparation}

Hawthorn (Lot No.180601) was purchased from Sichuan Tong-shan-tang TCM decoction pieces Co. Ltd and identified as dry mature fruit of Crataegus pinnatifida Bge by Professor Yan Zhiyong of Southwest Jiaotong University. A voucher specimen (ZJ00102516) was preserved at Zhejiang Institute of Food and Drug Inspection (Zhejiang, China). $100 \mathrm{~g}$ charred hawthorn $(\mathrm{CH})$ immersed in $400 \mathrm{~mL}$ distilled water for $30 \mathrm{~min}$ and extracted for $60 \mathrm{~min}$; and the $\mathrm{CH}$ residue was extracted with $200 \mathrm{~mL}$ distilled water for $30 \mathrm{~min}$. This extract mixture was filtered with gauze followed by concentrated via decompression, up to the concentration of $1 \mathrm{ml}$ decoction containing $0.3 \mathrm{~g} \mathrm{CH}$. Stir-frying HT referred to $2015 \mathrm{Chinese}$ Pharmacopoeia with fourth section. Drawing on the previous work ${ }^{[15]}$, an online non-contact temperature control system was make for the processing of $\mathrm{CHT}$ as follows: medium-fire at $380-450^{\circ} \mathrm{C}$; Stir-frying process at 8 minutes; yield at $90-94 \%$ (Supplementary Materials Table S2 and S3). The CHT decoction was prepared in the same way.

\subsection{Main chemical analysis in the stir-frying process of hawthorn}

Main ingredients of hawthorn were flavonoids and organic acids also including amino acids. Pharmacological studies showed that organic acid, amylase and protease play an important role in promoting digestion in hawthorn ${ }^{[16]}$, which implied the efficacy changes with these ingredients change in the pre and post stir-frying of hawthorn.

The content of organic acids in hawthorn was determined with reference to the acid-base titration method according to the Chinese pharmacopoeia (2015 edition). UV-vis spectrophotometry was used to detect the content of flavonoids with rut in as the reference substance at $520 \mathrm{~nm}$. Dinitrosalicylic acid (DNS) method and folin-phenol reagent method, both respectively coupled with UV-vis spectrophotometry to measure amylase activity with maltose as the reference substance at $540 \mathrm{~nm}$ and protease activity with tyrosine as the reference substance at $680 \mathrm{~nm}$. Amylase activity is expressed by the quality of maltose, produced by amylase of $1 \mathrm{~g}$ hawthorn samples catalyse-decomposing starch each minute. Amylase activity is expressed by the quality of maltose from amylase of $1 \mathrm{~g}$ hawthorn samples catalyse-decomposing starch each minute. Protease activity is expressed by the mass of tyrosine, produced by the enzyme of $1 \mathrm{~g}$ hawthorn samples catalysedecomposing the casein in 1 minute at $40^{\circ} \mathrm{C}$. Before the UV-vis spectrophotometry in the above experiments, methodological studies were performed, including linear relationship, the precsion test, stability experiment, repetitive experiment and recovery experiment, to ensure the rationality of the experimental conditions (Table S4-S7). 
Drawing on the previous work ${ }^{[17]}$, Hitachi L-8900 automatic amino acid analyzer was used to analyse and determine the types and content of amino acids in the stir-frying process of hawthorn. The spectra results were analyzed by the Ezchron of Hitachi company.

\subsection{Experimental design}

SPF grade Sprague-Dawley (SD) rats (170 $\pm 10 \mathrm{~g})$ were purchased from Dasheng Biotechnology Co., Ltd., (NO: SCXK (Chuan) 2018-0012). All procedures were done in accordance with the Guide for the Care and Use of Laboratory Animals (8th edition, National Academies Press), and with the approval of the Ethics Committee for Animal Experimentation of the Southwest Jiaotong University (see Supplementary Material). The rats were placed on clear animal house and allowed for food and water ad libitum under $12 \mathrm{~h}$ light/dark cycle at $22-24^{\circ} \mathrm{C}$ in 7-day adaptation period. The rats in control group with a normal diet, while the others fed with high-calorie diet (HCD) gavaged with $50 \%$ milk solution (10 mL/kg) for HC-DID modeling ${ }^{[18]}$. After successful modeling duration 10 days, these animals were randomly divided into the following six groups ( $\mathrm{n}=10)$ : model $(5 \mathrm{~g} / \mathrm{kg} / \mathrm{d}$ milk solution), cisapride $(2.7 \mathrm{mg} / \mathrm{kg} / \mathrm{d}), \mathrm{HT}$ (3 g/kg/d), CHT (3 g/kg/d), OCHT (10 g/kg/d water) and $\mathrm{CHT}+\mathrm{OCHT}(3 \mathrm{~g} / \mathrm{kg} / \mathrm{d})$. Aparting from rats in $\mathrm{OCHT}$ and $\mathrm{CHT}+\mathrm{OCHT}$ groups with treat with odor of charred hawthorn inhalation, the others inhaled clean air for $1 \mathrm{~h}$ once daily for 11 days via odor administration instrument (Patent number: 201810784238.6 and 201821130574.0 ) (Fig 1). Odor-inhalation administration methods were as follows: heating $300 \mathrm{~g}$ charred hawthorn to $45-50^{\circ} \mathrm{C}$ in the airtight chamber with the infrared thermometer monitor. The odor of charred hawthorn at a speed of $10 \mathrm{~L} / \mathrm{min}$ enter the animal room at $22^{\circ} \mathrm{C}$ using clean air as a carrier.

\subsection{Body weight and food intake in rats}

The body weight and food intake of rats were recorded every two days.

\subsection{Electroencephalography(EEG)}

Twenty rats in control and model groups were placed on the $37^{\circ} \mathrm{C}$ thermostatic rat plate under anesthesia with intraperitoneal injection of $10 \%$ chloral hydrate $(3 \mathrm{mg} / \mathrm{kg})$. Electrophysiologic surgical procedure was performed in a quiet room without electromagnetic interference. The skulls of rats were fixed and exposed on the brain stereotaxic apparatus (RWD Life Science Co., Ltd., Shenzhen, China) followed by the localization of detecting electroencephalography (EEG) area which including hypothalamus, olfactory bulb and the ventral nucleus of lateral lemniscus with the help of the rat brain atlas (George Paxinos \&Charles Watson, 5th edition), where drilling holes in these area at low speed, physiological saline cooling to reduce the stimulation. The burr holes were made for insertion of $0.25-\mathrm{mm}$ diameter acupuncture pins electrodes with no conductivity besides EEG recording area. Three electrodes were insertion respectively in VMHC of the hypothalamus (coordinates: anterior-posterior $[\mathrm{AP}]=-2.56 \mathrm{~mm}$; medial-lateral $[\mathrm{ML}]=0.6 \mathrm{~mm}$; dorsal-ventral $[\mathrm{DV}]=9.7 \mathrm{~mm}$ ); AOL of the olfactorybulb (coordinates: $A P=5.2 \mathrm{~mm} ; \mathrm{ML}=2.2 \mathrm{~mm} ; \mathrm{DV}=5.8 \mathrm{~mm}$ ); VLLof the ventral nucleus of lateral lemniscus as reference electrodes (coordinates: $A P=-8 \mathrm{~mm} ; \mathrm{ML}=2.4 \mathrm{~mm} ; \mathrm{DV}=8.4 \mathrm{~mm}$ ). They were fixed in place with dental cement. The ground electrode was inserted into the left hamstring muscle of the rats to the depth of $2 \mathrm{~cm}$. The stimulus consists of odor of charred hawthorn and clean air alternating at one-minute interval, with specific conditions on odor temperature at $36^{\circ} \mathrm{C}$, humidity at $80 \%$, nasal inhalation. EEG data were recorded wide-band $0.1 \mathrm{~Hz}$ to $5.0 \mathrm{kHz}$, sampled at $1 \mathrm{kHz} / \mathrm{channel}(4$ channels); 200 ms time constant using BL-420i biological function experiment system from Chengdu Taimeng software Co., Ltd. (Chengdu, China), displayed and then stored on computer duration of $40 \mathrm{~min}$.

\subsection{Electrogastrography (EGG)}

After $18 \mathrm{~h}$ fast and $2 \mathrm{~h}$ water deprivation, 42 rats in 7 groups $(\mathrm{n}=6)$ were operated to midline laparotomy on $37^{\circ} \mathrm{C}$ thermostatic rat plate where inhalation anesthesia with $1.5-2.0 \%$ isoflurane. One pair of $\mathrm{Ag} / \mathrm{AgCl}$ electrode was punctured in the serosal layer of the gastric antrum for about $1 \mathrm{~cm}$ at $2 \mathrm{~cm}$ interval allows for the interference of electrical signals between them. The ground electrode was used to clampe the skin of the rats, abdomen. Electrogastrography (EGG) operation was conducted in the serosal layer of the duodenum of rats with the same process. The BL-420i bio-functional 
system recorded the electrical signals of the gastrointestinal tract of rats for 40 min (parameters: Gain [G] = $1.0 \mathrm{mv}$; time constant $[\mathrm{T}]=200 \mathrm{~ms}$; filtering $[\mathrm{F}]=1 \mathrm{KHz}$ ). The continuous amplitudeandfrequencyofEGGwereanalyzedifitwasstabilizedwithin10min.

\subsection{Experiment of gastric emptying and intestinal propulsion}

Gastric emptying and small intestinal propulsion experiments were performed in accordance with existing studies ${ }^{[19]}$. After an overnight fast, each rat in all groups was administered as usual in line with experimental design in Materials and methods. After 30 minutes, all rats were intragastric administration with $4 \mathrm{~mL}$ nutritive semi-solid paste, which consisted of $10 \mathrm{~g}$ sodium carboxymethylcellulose, $16 \mathrm{~g}$ milk powder, $8 \mathrm{~g}$ starch, $8 \mathrm{~g}$ sugar and $2 \mathrm{~g}$ activated charcoal in all $300 \mathrm{~mL}$ semisolid paste, and then sacrificed after $20 \mathrm{~min}$. With reference to the relevant literature [19], the gastric emptying rate and small intestinal propulsion rate were as follows:

\section{Gastric emptying rate $=(A-B) / A * 100 \%$}

\section{Small intestinal propulsion rate $=X / Y * 100 \%$}

A: semi-solid paste quality.

B: gastric residue quality

X: advanced length of semi-solid paste.

Y: total length of the small intestine

\subsection{Test of digestive juice in rats}

Gastric juice of each rat in all groups were collected with centrifugal tube, at $4000 \mathrm{r} / \mathrm{min}$ for $5 \mathrm{~min}$ followed by recorded the volumes. The gastric juice acidity was determined using acid-base titration and the peps in activity was performed according to the method described by Jiang et al. [19]. Two freshly protein tubes with Mettcapillary method prepared, were placed into $50 \mathrm{~mL}$ trig flask containing the mixture of $1 \mathrm{~mL}$ gastric juice and $15 \mathrm{~mL} 50 \mathrm{mmol} / \mathrm{L}$ hydrochloric acid with an incubator at $37^{\circ} \mathrm{C}$ for $24 \mathrm{~h}$, and then the average length of the transparent part at both ends of the protein tube ( $\left.\mathrm{mm}\right)$ was measured with a vernier caliper. With reference to the relevant literature ${ }^{[19]}$, the total gastric acid flux and the pepsin activity were as follows:

\section{Total gastric acid flux $=M * N$}

\section{Pepsin activity $=\mathrm{Y}^{2} * 16$}

M: the gastric juice acidity

$\mathrm{N}$ : the volume of gastric juice

Y: the average length of the transparent part of the protein tube

\subsection{Samples collection}

Rats were fasted for $12 \mathrm{~h}$. Ten percent chloral anaesthesia $(3.5 \mathrm{~mL} / \mathrm{kg})$ was used as an anesthetic agent. Blood samples were collected from abdominal aorta of rats after anaesthesia. Serum separator tubes were used to collect and allowed to clot for 2 hours at room temperature. Serum was removed and stored at $-80^{\circ} \mathrm{C}$ after the blood has been centrifuged. Some gastric antrum, duodenum and hypothalamus were immediately removed from an environment with a low temperature at stored in liquid nitrogen. Another were fixed in a $10 \%$ neutral formalin solution, embedded in paraffin, and preserved at $-80{ }^{\circ} \mathrm{C}$ 


\subsection{Pathological examination}

After $12 \mathrm{~h}$ fast, rats in all group were sacrificed and then separated quickly hypothalamus, gastric antrum and duodenum. These tissue were rinsed with physiological saline, fixed with freshly formulated $4 \%$ paraformaldehyde, cutted into 4 -umthick paraffin sections with RM-2016 rotary microtome (Leica, Germany) that were stained with H\&E staining. Routine histopathological examination was performed under trinocular digital microscope (BA400Digital『MOTIC区China).

\subsection{Elisa analysis}

Rat ELISA SCF kit (ZC-36620, ZCiBio, China) was used to detect the serum level of corresponding index via MultlskanMk3 enzyme-labeling instrument (Thermo Fisher Scientific, Germany). The experimental procedure follows the manufacturer instructions.

\subsection{Immunohistochemistry analysis}

The tissues, fixed with $4 \%$ paraformaldehyde, were dehydrated, embedded and cut by conventional procedures. The sections were treated with $3 \%$ methanol peroxide for $10 \mathrm{~min}$, washed with $0.1 \mathrm{~mol} / \mathrm{L}$ phosphate buffer saline (PBS) (pH 7.2 7.4凶for 3times, and then put into $0.01 \mathrm{M}$ citrate buffer $(\mathrm{pH} 6.0)$ by heating for antigen rest orationd. The sections were blocked with normal goat serum (ZLI-9021, ZSJQ-Bio Co., Ltd., Beijing, China) for 20 min followed by primary antibody (SCF: gxp285443, Gen Xspan, Inc. Alabama, USA; c-Kit: ab25022, Abcam, UK) incubation for $12 \mathrm{~h}$ at $4{ }^{\circ} \mathrm{C}$, and then in cubation with secondary biotinylated antibody (SP-9001, ZSJQ-BioCo., Ltd. Beijing, China) for $0.5 \mathrm{~h}$ at $37^{\circ} \mathrm{C}$. After rinsing with PBS for 3 time every 5 min, using L-2,4-Diaminobutyrate(DAB) (K135925C, ZSJQ-Bio Co., Ltd., Beijing, China) as chromogenic reagent for 2 min, and then washing sections with distilled water. After counterstaining sections with hematoxylin, image of the sections was captured (100X and 400X) by BA200Digita microphotography system (Motic, China), respectively, conducted in triple. Integrated optical density (IOD) value of all images were measured by image-pro Plus 6.0 image analysis system (Media Cybernetics, Inc. USA).

\subsection{Reverse transcription polymerase chain reaction (RT-PCR) analysis}

Total RNA was isolated from gastric antrum homogenate using Trizol reagent (Invitrogen, USA). After genomic DNA elimination, reverse transcription was performed using PrimeScript RT reagent Kit (Dalian, China). Gene-specific primers were screened using Primer Premier design software, based on National Center for Biotechnology Information (NCBI) databases, and then synthesized at bioengineering technology Co. (Shanghai, China). Primers and base sequences used in this study are shown in Table 1. Quantitative RT-PCR was accomplished by PIKORed 96 PCR instrument (Thermo Fisher, USA) coupled with Thermo Scientific PikoReal software. The relative expression lever of the gene was calculated by the threshold cycle (CT) value and the fold increase over control $\left(2^{-\triangle \Delta C T}\right)$.

Table 1 Primer nucleotide sequences

\begin{tabular}{|lll|}
\hline Genes & Primers & Nucleotide sequences 5'-3' \\
SCF & Forward & GTGGATGACCTCGTGGCATGTATGGA \\
\cline { 2 - 3 } C-kit & Reverse & CCACCATGAAGTCCTTGAAGGCATCA \\
& Forward & GCTGCTCATTGGCTTCGTGGTCACA \\
\cline { 2 - 3 } B-actin & Reverse & ACCGTCATGGCGGCATCCGACTTAA \\
& Ferwerse & TACAGATCAAGATCATTGCTCCT \\
& ReVTTGCTGATCCA \\
\hline
\end{tabular}

\subsection{Statistical analysis}


Student T tests was performed to compare the means of two groups. Statistical analysis was performed compare the means of 3 or more groups by One-way ANOVA (or Kruskal Walis test). Data are expressed as mean \pm SD if normally distributed. SPSS 20.0 was used for all statistical analyses. $P<0.05$ was considered significant.

\section{Results}

\subsection{Main chemical analysis in the stir-frying process of hawthorn}

As shown in table 2, the content of organic acid, amylase activity and protease activity in the charred hawthorn were decreased by $26.12 \%, 19.38 \%$ and $28.05 \%$, respectively, as compared with the raw hawthorn, suggesting that effective components for promoting digestion were significantly decreased after the stir-frying process of the hawthorn. After the stirfrying process of the hawthorn, the content of flavonoids increased by $12.91 \%$ (Table 2), while there was no change in the content of amino acids. There are 21 types of amino acids (Table S8), including 18 types of common amino acids (except tryptophan that was destroyed by acid hydrolysis) in fried charred process of hawthorn. And there are 8 essential amino acids in human body, respectively threonine, valine, leucine, isoleucine, methionine, phenylalanine, lysine, tryptophan, in addition, also measured 3 kinds of amino acids, are the ornithine, hydroxyproline and gamma aminobutyric acid, this is consistent with the results of the pieces of amino acids in hawthorn fruit determined by Ainian Guan, XiaLiu [20,21].

Table 2 Main components changes before and after frying of hawthorn

\begin{tabular}{|lll|}
\hline Components & Raw hawthorn & Charred hawthorn \\
\hline Organic acids $(\mathrm{mg} / \mathrm{g})$ & 74.89 & 55.33 \\
\hline Flavonoids $(\mathrm{mg} / \mathrm{g})$ & 51.21 & 58.80 \\
\hline Amino acids $(\mathrm{mg} / \mathrm{g})$ & 6.73 & 6.81 \\
\hline Amylase activity $(\mathrm{mg} / \mathrm{min} / \mathrm{g})$ & 2.53 & 2.04 \\
\hline Protease activity $(\mathrm{mg} / \mathrm{min} / \mathrm{g})$ & 0.82 & 0.59 \\
\hline
\end{tabular}

\subsection{Body weight changes}

As shown in table 3, there was no significant difference among the rats of all groups in the initial body weight. After successful modeling, the body weight and food intake in rats with diet-induced dyspepsia were significantly reduced compared with that of the control group with normal diet. After treatment with cisapride, $\mathrm{HT}, \mathrm{CHT}, \mathrm{OCHT}$ and $\mathrm{CHT}+\mathrm{OCHT}$, body weight and food intake of each group began to recover in different degrees, and the $\mathrm{CHT}+\mathrm{OCHT}$ group had better effects.

Table 3 Changes of body weight and food intake 


\begin{tabular}{|lllllll|}
\hline Group & \multicolumn{5}{l}{ Body weight } & \multicolumn{5}{l|}{ Food intake } \\
\cline { 2 - 7 } & $\mathrm{a}$ & $\mathrm{b}$ & $\mathrm{c}$ & $\mathrm{a}$ & $\mathrm{b}$ & $\mathrm{c}$ \\
\hline Control & $191.4 \pm 9.2$ & $229.5 \pm 10.5$ & $269.7 \pm 13.0$ & $32.71 \pm 3.23$ & $34.12 \pm 2.76$ & $35.18 \pm 2.32$ \\
\hline Model & $201.3 \pm 5.5$ & $221.0 \pm 9.2$ & $234.9 \pm 15.0$ & $22.32 \pm 2.05$ & $18.5 \pm 2.01$ & $21.18 \pm 2.41$ \\
\hline Cisapride & $202.0 \pm 13.8$ & $223.7 \pm 9.5$ & $268.6 \pm 12.7$ & $23.04 \pm 2.31$ & $19.43 \pm 1.45$ & $25.46 \pm 2.44$ \\
\hline HT & $200.5 \pm 9.1$ & $218.6 \pm 9.1$ & $248.4 \pm 11.6$ & $23.62 \pm 1.34$ & $19.32 \pm 2.22$ & $24.38 \pm 2.07$ \\
\hline CHT & $206.1 \pm 14.6$ & $225.6 \pm 12.1$ & $243.9 \pm 11.2$ & $24.9 \pm 2.09$ & $19.51 \pm 1.68$ & $25.41 \pm 1.36$ \\
\hline OCHT & $202.2 \pm 17.2$ & $223.9 \pm 9.5$ & $260.6 \pm 12.5$ & $23.74 \pm 2.22$ & $18.25 \pm 1.85$ & $27.23 \pm 1.79$ \\
\hline CHT+OCHT & $207.5 \pm 13.8$ & $228.9 \pm 20.8$ & $282.7 \pm 17.9$ & $25.38 \pm 2.47$ & $16.83 \pm 1.68$ & $27.81 \pm 2.23$ \\
\hline
\end{tabular}

"a" means before molding; "b" means after molding; "c" means after dosing.

\subsection{Electroencephalography(EEG)}

The results are shown in figure 2. VMHC area of the hypothalamus and $\mathrm{AOL}$ area of the olfactory bulb in control rats that were given $\mathrm{OCHT}$, the amplitude increased by $8.3 \%$ and $3.4 \%$ on average, and the frequency decreased averagely by $16 \%$ and $8.6 \%$, compared with that of rats given clean air. VMHC area of the hypothalamus and AOL area of the olfactory bulb in model rats that were given $\mathrm{OCHT}$, the amplitude increased by $3.40 \%$ and $5.10 \%$ on average, and the frequency decreased averagely by $25.8 \%$ and $7.40 \%$, compared with that of rats given clean air.

\subsection{Electrogastrography (EGG)}

The results are shown in figure 3 . The amplitude in the gastric antrum and duodenum of the model rats was significantly decreased $(P<0.01)$; The frequency in the gastric antrum significantly decreased $(P<0.05)$ and that of duodenum was no significance $(P>0.05)$, compared with that of the control rats. Compared with the model group, each treatment group (cisapride, $\mathrm{HT}, \mathrm{CHT}, \mathrm{OCHT}$ and $\mathrm{CHT}+\mathrm{OCHT}$ ) had no significant effect on the frequency in the gastric antrum and duodenum of the rats, but they excepting for the smell group enhanced amplitude in these rats $(P<0.01$ or $P<0.01)$ (therapeutic effect: Cisapride $>\mathrm{OCHT}+\mathrm{CHT}>\mathrm{HT}>\mathrm{CHT}>\mathrm{OCHT}$ ). Notably, the odor of charred hawthorn could enhance gastric smooth muscle contraction and a combination of charred hawthorn decoction and odor of charred hawthorn had better effect.

\subsection{Gastrointestinal motility and digestive juice secretion}

The results are shown in table 4. Compared with the control group, gastric emptying rates and intestinal propulsion rates in diet-induced dyspepsia rats was significantly reduced $(P<0.05)$. Compared with the model group, each treatment group (cisapride, $\mathrm{HT}, \mathrm{CHT}, \mathrm{OCHT}$ and $\mathrm{CHT}+\mathrm{OCHT}$ ) improved gastrointestinal motility of dyspepsia rats in different degrees, with significant differences $(P<0.05, P<0.01$ or $P<0.001)$ (therapeutic effect for gastric emptying function: $\mathrm{OCHT}+\mathrm{CHT}>\mathrm{HT}>\mathrm{CHT}$ $>\mathrm{OCHT}>$ Cisapride; therapeutic effect for intestinal propulsion ability: $\mathrm{OCHT}+\mathrm{CHT}>\mathrm{CHT}>$ Cisapride $>\mathrm{OCHT}$ group $>\mathrm{HT}$ group). Notably, the odor of charred hawthorn regulated gastrointestinal function and a combination of charred hawthorn decoction and odor of charred hawthorn works best.

Therefore, we further investigated the effect of drug treatment on digestive juices of rats, including gastric juice content, gastric acidity, total gastric acid flow and pepsin activity (Table 4). Compared with the control group, gastric juice secretion, total gastric acid flow decreased and gastric acidity decreased in the model group $(P<0.01 ; P<0.001 ; P<0.05)$, pepsin activity significantly increased $(P<0.001)$. The rats treated with cisapride, $\mathrm{HT}, \mathrm{CHT}, \mathrm{OCHT}$ and $\mathrm{CHT}+\mathrm{OCHT}$, had different degree in improve the digestive juice secretion compared with the normal rats. It is worth noting that the OCHT significantly increased the total gastric acid flow and pepsin activity, and a combination of CHT and OCHT had better effect. 
Table 4 Gastrointestinal motility and digestive juice secretion

\begin{tabular}{|c|c|c|c|c|c|c|}
\hline Group & $\begin{array}{l}\text { Gastric } \\
\text { emptying } \\
\text { rate(\%) }\end{array}$ & $\begin{array}{l}\text { Intestinal } \\
\text { propulsion } \\
\text { rate(\%) }\end{array}$ & $\begin{array}{l}\text { Gastric juice } \\
\text { content }(\mathrm{mL})\end{array}$ & $\begin{array}{l}\text { Gastric } \\
\text { acidity } \\
(\mathrm{mmol} / \mathrm{L})\end{array}$ & $\begin{array}{l}\text { Total gastric } \\
\text { acid flow } \\
(\mu \mathrm{moL} / 4 \mathrm{~h})\end{array}$ & $\begin{array}{l}\text { Pepsin } \\
\text { activity } \\
(\mathrm{U} / \mathrm{mL})\end{array}$ \\
\hline Control & $84.50 \pm 6.71 * \star \star *$ & $76.80 \pm 14.22^{\star \star \star}$ & $4.62 \pm 1.02^{\star \star}$ & $52.19 \pm 8.44$ & $238.59 \pm 42.39 * \star \star$ & $167.4 \pm 29.1$ \\
\hline Model & $58.00 \pm 15.55$ & $55.90 \pm 3.09$ & $3.32 \pm 0.87$ & $50.12 \pm 8.28$ & $163.22 \pm 27.49$ & $212.4 \pm 22.8$ \\
\hline Cisapride & $74.00 \pm 9.78^{*}$ & $70.99 \pm 4.55^{\star \star}$ & $5.37 \pm 1.11$ *** & $59.62 \pm 10.23^{*}$ & $312.15 \pm 39.71$ *** & $329.8 \pm 31.3^{\star \star \star}$ \\
\hline HT & $81.00 \pm 11.40 * *$ & $66.32 \pm 3.06 * \star \star$ & $3.88 \pm 0.73$ & $59.49 \pm 6.86 *$ & $234.77 \pm 28.36^{\star \star \star}$ & $251.3 \pm 36.6 *$ \\
\hline $\mathrm{CHT}$ & $74.12 \pm 10.84^{\star}$ & $72.63 \pm 2.28 * \star \star$ & $4.82 \pm 0.94^{\star \star}$ & $58.38 \pm 11.91$ & $280.16 \pm 27.18^{\star \star \star}$ & $303.2 \pm 25.4^{\star \star \star}$ \\
\hline OCHT & $79.00 \pm 13.76$ ** & $69.32 \pm 3.52^{\star \star}$ & $4.07 \pm 0.92$ & $57.10 \pm 9.03$ & $235.56 \pm 21.94^{\star \star \star}$ & $262.7 \pm 36.2^{\star \star}$ \\
\hline $\mathrm{CHT}+\mathrm{OCHT}$ & $81.50 \pm 8.22^{\star \star}$ & $74.44 \pm 7.98 * \star \star$ & $5.34 \pm 1.20 * \star \star$ & $65.75 \pm 11.4^{\star *}$ & $338.15 \pm 31.70 * \star \star$ & $368.8 \pm 29.1^{\star \star *}$ \\
\hline
\end{tabular}

$\mathrm{HT}$ : hawthorn group, $\mathrm{CHT}$ : charred hawthorn group, OCHT: odor of charred hawthorn, $\mathrm{CHT}+\mathrm{OCHT}$ : charred hawthorn + odor of charred hawthorn group. Data were analyzed by one-way ANOVA (mean \pm SD, $n=6$ ).SPSS 17.0 was used for statistical analyses. VS model group, ${ }^{*} P<0.05, * \star P<0.01$.

\subsection{Histopathological examination}

It can be seen from Figure 4, there were no pathological changes in hypothalamus, gastric antrum and duodenum tissue by HE staining.

\subsection{SCF/c-Kit pathway}

Because of SCF/c-Kit pathways can regulate gastrointestinal motility, we further study level of the relevant indexes including SCF and c-Kit in all rats (Fig. 5). Compared with control group, HCD caused SCF/c-Kit pathway disorder of model group, serum level and mRNA expression as well as protein expression of these indexes significantly decreased. HT, CHT and $\mathrm{CHT}+\mathrm{OCHT}$ could regulate the SCF/c-kit pathway in the rats with diet-induced dyspepsia to the normal closely level (therapeutic effect: $\mathrm{CHT}>\mathrm{OCHT}+\mathrm{CHT}>\mathrm{HT}$ ). Notably, SCF and c-kit levels in the OCHT group were higher than that of the model group, but there was no significant difference.

\section{Discussion}

Because of "Shi Ji" in traditional Chinese medicine (TCM) is similar to FD in western medicine, the "Shi Ji" model rats were prepared by using high-calorie diet intervention (TCM etiology) combined with physical signs and intestinal pathology verification (western medicine pathology). The HC-DID rats showed the loss of body weight and food intake, gastrointestinal motility decreased and changes of the physical signs, all of which are indicative of the suitability of the model to be used in researching the effects of hawthorn on experimental HC-DID in rats ${ }^{[20]}$.

Stir-baking is a unique and effective processing method summarized from the clinical practice of TCM, Stir-baking products is believed to treat digestive diseases ${ }^{[21-23]}$. The Chinese pharmacopoeia stipulate the characteristics of the charred hawthorn, but no specific processing technology. Therefore, it is the basis and key to obtain charred hawthorn possessing stable quality and uniform color as well as the charred odor. According to Chinese Pharmacopoeia, the non-contact infrared the monitor is used to collect temperature data during the stir-frying process, combined with the traditional heat description of "Low fire, Middle fire, High fire". Table 1 and Table 2 show the samples of stir-frying process. The measured corresponding to the scope of the temperature through pre-experiment, gets processed products in three different levels of 
heat: Low fire, Middle fire, High fire. The raw hawthorn is numbered SZO. And the samples of stir-frying process are shown in Fig. 1. The results showed that the charred hawthorn obtained by frying $150 \mathrm{~g}$ raw hawthorn under the condition of moderate fire for $10 \mathrm{~min}$ at $380-420^{\circ} \mathrm{C}$, could be used for further experimental study.

Our study proved that charred hawthorn is more effective in digestion than raw hawthorn, but paradoxically the active components (organic acids and digestive enzymes) related to digestion in charred hawthorn are significantly reduced. The obvious change of odor maybe an important reason why the effect of $\mathrm{CHT}$ on digestion is better than that of HT. Through headspace solid-phase micro-extraction gas chromatography-mass spectrometry (HS-SPME-GC/MS) analysis, the contents of 11 main volatile substances (odors) of charred hawthorn were significantly higher than that of raw hawthorn. In addition, 3-methylbutyraldehyde, 2-methylbutyraldehyde, 5-Hydroxymenthyl-2-furfural (5-HMF) and furfural have been found to have digestive effects ${ }^{[13-15]}$. Therefore, we speculate that volatile substances (odor) in the charred hawthorn may promote digestion.

Olfactory and brain have extensive connection, when odor activates olfactory receptor, followed by send out electric signal to brain through nerve axon, thereby regulating physiological activities and behavior ${ }^{[24,25]}$. Odor administration as central nervous system agents, may be a new way directly acting on the brain. Aromatic Chinese medicine or foods are mainly used to treat depression, alzheimer's disease, Parkinson's disease and immune function via olfactory pathway, but little research has been done on the effect of charred odor in the stir-frying process of charred hawthorn on diet-induced dyspepsia [26-29]. The odor of $\mathrm{CHT}$ is difficult to be administrated to animal and human because it is easily dissipated once stop heating the $\mathrm{HT}$. Dose of the odor is also an issue. In light of that, we developed an instrument using for odor administration. The animal experiment showed that digestion effect of the odor is the best by observing the changes in body weight and food intake, gastrointestinal motility experiment, with a condition of when the heat temperature at $45-50{ }^{\circ} \mathrm{C}, 10 \mathrm{~L} / \mathrm{min}$ flow rate, $1 \mathrm{~h}$ odor administration time. Olfactory bulb is the physiological basis of odor administration, and hypothalamus is the integrated center of energy balance and regulation of gastrointestinal function. Since EEG is an intuitive reflection of electrical activity, excitation or inhibition in brain regions, we further investigated the effects of $\mathrm{OCHT}$ on that of these regions using EEG [30,

31]. Raw hawthorn and charred hawthorn can significantly increase the amplitude of isolated gastrointestinal smooth muscle in rats. EEG was an important tool in the study of gastrointestinal dynamics, recording the gastrointestinal slow wave rhythm, including frequency and amplitude. Our experiment proved that OCHT may had excitant effect on the hypothalamus and olfactory of rats. SCF/c-Kit pathway can regulate gastrointestinal smooth muscle contraction and gastrointestinal motility depending on play a part in ICC. Our further study demonstrated that hawthorn decoction (raw hawthorn and charred hawthorn) could treat diet-induced dyspepsia in rats by regulating signaling pathways, a combination of charred hawthorn decoction and odor of charred hawthorn works best. Notably, OCHT could increase SCF and c-kit levels in the model rats, but there was no significant difference.

\section{Conclusion}

Our study proved that charred hawthorn is more effective in digestion than raw hawthorn, but paradoxically the digestiverelated components in charred hawthorn are significantly reduced. Based on HS-SPME-GC/MS analysis between OCHT and HT in our previous study, we further explored OCHT affects the brain (central nervous system) of rats. Consistently, symptoms of HC-DID was improved in rats after 10-day oral administration of hawthorn decoction (raw hawthorn and charred hawthorn), interestingly, OCHT also had good curative effect for it after 10-day odor treatment. The effect of hawthorn decoction on HC-DID in rats by modulating SCF/c-kit pathway, but the action mechanism of OCHT still needs further study.

\section{Declarations}

\section{Ethics approval and consent to participate}


All procedures were done in accordance with the Guide for the Care and Use of Laboratory Animals (8th edition, National Academies Press), and with the approval of the Ethics Committee for Animal Experimentation of the Southwest Jiaotong University (see Supplementary Material).

\section{Consent for publication}

Not applicable.

\section{Availability of data and materials}

The datasets used and/or analyzed during the current study are available from the corresponding author on reasonable request.

\section{Competing interests}

The authors declare no competing financial interest.

\section{Funding}

This work was supported by National Natural Science Foundation of China (No. 81603295), which was used for experimental design, Science \& Technology Department of Sichuan Province (No. 2020YJ0100), which was used for collection and analysis of data, and China Scholarship Council (No. 201807005061), which was used to purchase raw materials and reagents.

\section{Acknowledgements}

Not applicable.

\section{Authors contributions}

Q He, J Wan, Y Wang, and X Zhou: experiment design; Q He, C Liu and T Wang: animal experiments; JY Sun and C Liu: preparation of medicinal materials and chemical composition analysis of hawthorn; Q He, T Wang, C Liu and L Ma: the data analysis; Q He, T Wang and J Wan: the whole experiment process and manuscript writing. All authors read and approved the manuscript, and all data were generated in-house and that no paper mill was used.

\section{References}

1. Bai C, Zheng ZA, Xu JN, et al. Establishment and evaluation of dyspeptic (Shi Ji) model animals with accumulated heat in stomatch and intestine[J]. J Hunan Univ Chin Med, 2018, 2:125-129.

2. Bian L, Dou ZH. Effect of aqueous extract from Dahuang (Rhei Radix et Rhizoma) on gastrointestinal movement, serum motilin and gastrin levels of dyspepsia mice model[J]. J Gansu Coll Tradit Chin Med. 2018, 35(05):26-29.

3. Wang Y F, Gao WY, Xu X, et al. Effect of massa medicate fermentata composed by fresh or dry Chinese medicines on gastrointestinal motility and intestinal flora regulating of dyspepsia mice[J]. Chinese Journal of Experimental Traditional Medical Formulae, 2017, 23(4):20-24.

4. Liu J, Li F, Tang XD, et al. Modern clinical studies on TCM syndrome of functional dyspepsia and their differentiation standards[J]. World Chinese Medicine, 2015, 10(1):56-59.

5. Tang Y, Tang MW, LOU QX, et al. Advances in TCM Treatment of Functional Dyspepsia[J]. China Journal of Chinese Medicine, 2020, 35(2):299-303.

6. Bashamboo A, Taylor AH, Samuel K, et al. The survival of differentiating embryonic stem cells is dependent on the SCFkit pathway[J]. J Cell Sci, 2006, 119(15):3039-3046. 
7. Thomsen L, Robinson TL, Lee JC, et al. Interstitial cells of Cajal generate a rhythmic pacemaker current[J]. Acta Physiol (Oxf). 1998, 187(3):391-397.

8. Szucs KF, Nagy A, Grosz G, et al. Correlation between slow-wave myoelectric signals and mechanical contractions in the gastrointestinal tract: Advanced electromyographic method in rats[J]. Journal of Pharmacological and Toxicological Methods, 2016, 82:37-44.

9. Chen Y, Xu J, Liu S, et al. Investigation of autophagy and differentiation of myenteric interstitial cells of Cajal in the pathogenesis of gastric motility disorders in rats with functional dyspepsia[J]. Biotechnol Appl Biochem, 2013, 41(6):1233-1249.

10. Wu J, Peng W, Qin R, et al. Crataegus pinnatifida: chemical constituents, pharmacology, and potential applications[J]. Molecules, 2014,19(2):1685-1712.

11. Wu JH, Sun JY. Effect of organic acid of hawthorn on gastrointestinal motility[J]. Shanxi TCM, 2009, 30(10):1402-1403.

12. State pharmacopoeia committee. Pharmacopoeia of the Peoples Republic of China (2015). Vol 1 [S]. $2005: 31$.

13. Zhou YQ, He FY, Yang YT, et al. Research situation of Maillard reaction and its influence on research methods for processing and preparation process of Chinese materia medica[J]. Chinese Traditional and Herbal Drugs, 2014, 45(1):125-130.

14. Xu Y. Study on the "coke aroma" material basis that promote digestion and the synergistic mechanism of "Jiao San Xian" after charred[D]. Southwest JiaoTong University, 2018.

15. Zhong L, Wang YW, Peng W, et al. Headspace solid-phase microextraction coupled with gas chromatography-mass spectrometric analysis of volatile components of raw and stir-fried fruit of C.Pinnatifida (FCP)[J]. Tropical Journal of Pharmaceutical Research, 2015, 14(5):1319-1326.

16. Yu BB, Yan XS, Sun DD. Research progress in pharmacological activities and mechanism of hawthorn[J]. Central South Pharmacy,2015,13(7): 745-748.

17. Zhan Y, Wan J, Zhou X. Effect of coking on the contents of amino acids in hawthorn[J]. Amino Acids and Biotic Resources, 2013, 35(3):28-31.

18. Xing JF, Feng WY, Hou JY. Methods of gastric emptying and intestinal propulsion in mice[J]. Journal of Beijing University of Traditional Chinese Medicine. 2003, 26(4):50-52.

19. Jiang T, Tang CP, Chen YF, et al. Effects of total flavonoids from galangal on experimental gastric ulcer in rats[J]. Journal of Chinese Medicinal Materials, 2009, 32(2):260-262.

20. Pu BQ, Zhang XN, Li DX, et al. Study on the Dynamic Changes of Free Amino Acids in Crataegus pinnatifida During Ripening[J]. Guangzhou Chemical Industry, 2020, 48(10):105-107.

21. Liu X, Ma XJ. Analysis and determination on the inorganic elements and amino acids in Hawthorn[J]. Chinese Wild Plant Resources, 1998, 17(2):37-39.

22. Peng W, Liu, YJ, Wu, N, et al. Areca catechu L. (Arecaceae): a review of its traditional uses, botany, phytochemistry, pharmacology and toxicology[J]. Journal of Ethnopharmacology, 2015, 164, 340-356.

23. Zhang SY, Sun GX, Feng B, et al. Study on the effect of different processed betel nut on gastrointestinal function[J]. Yunnan Journal of Traditional Chinese Medicine and Materia Medica, 2010, 31:50-52.

24. Bienenstock J, Kunze WA, Forsythe P. Disruptive physiology: olfaction and the microbiome-gut-brain axis[J]. Biol Rev Camb Philos Soc, 2018, 93(1):390-403.

25. Reisert J, Lai J, Yau KW, et al. Mechanism of the excitatory Cl- response in mouse olfactory receptor neurons[J]. Neuron. 2005, 45(4):553-561.

26. Komori T, Fujiwara R, Tanida M, et al. Effects of citrus fragrance on immune function and depressive states[J]. Neuroimmunomodulation, 1995, 2(3):174-80.

27. Matsuura T, Yamaguchi T, Zaike Y, et al. Reduction of the chronic stress response by inhalation of hiba (Thujopsis dolabrata) essential oil in rats[J]. Biosci Biotechnol Biochem, 2014, 78(7):1135-1139. 
28. Liang M, Du Y, Li W, et al. SuHeXiang essential oil inhalation produces antidepressant and anxiolytic-like effects in adult mice[J]. Biol Pharm Bull, 2018, 41(7):1040-1048.

29. Yoshifumi Irie. Effects of eugenol on the central nervous system: its possible application to treatment of Alzheimer's disease, depression, and parkinson's disease[J]. Current Bioactive Compound, 2006, 2(1):57-66.

30. Lundt A, Wormuth C, Siwek ME, et al. EEG radiotelemetry in small laboratory rodents: a powerful state-of-the art approach in neuropsychiatric, neurodegenerative, and epilepsy research[J]. Neural Plasticity. 2015, 2015:1-19.

31. Medlej Y, Asdikian R, Wadi L, et al. Enhanced setup for wired continuous long-term EEG monitoring in juvenile and adult rats: application for epilepsy and other disorders[J]. BMC Neuroscience, 2019, 20(1):8.

\section{Figures}
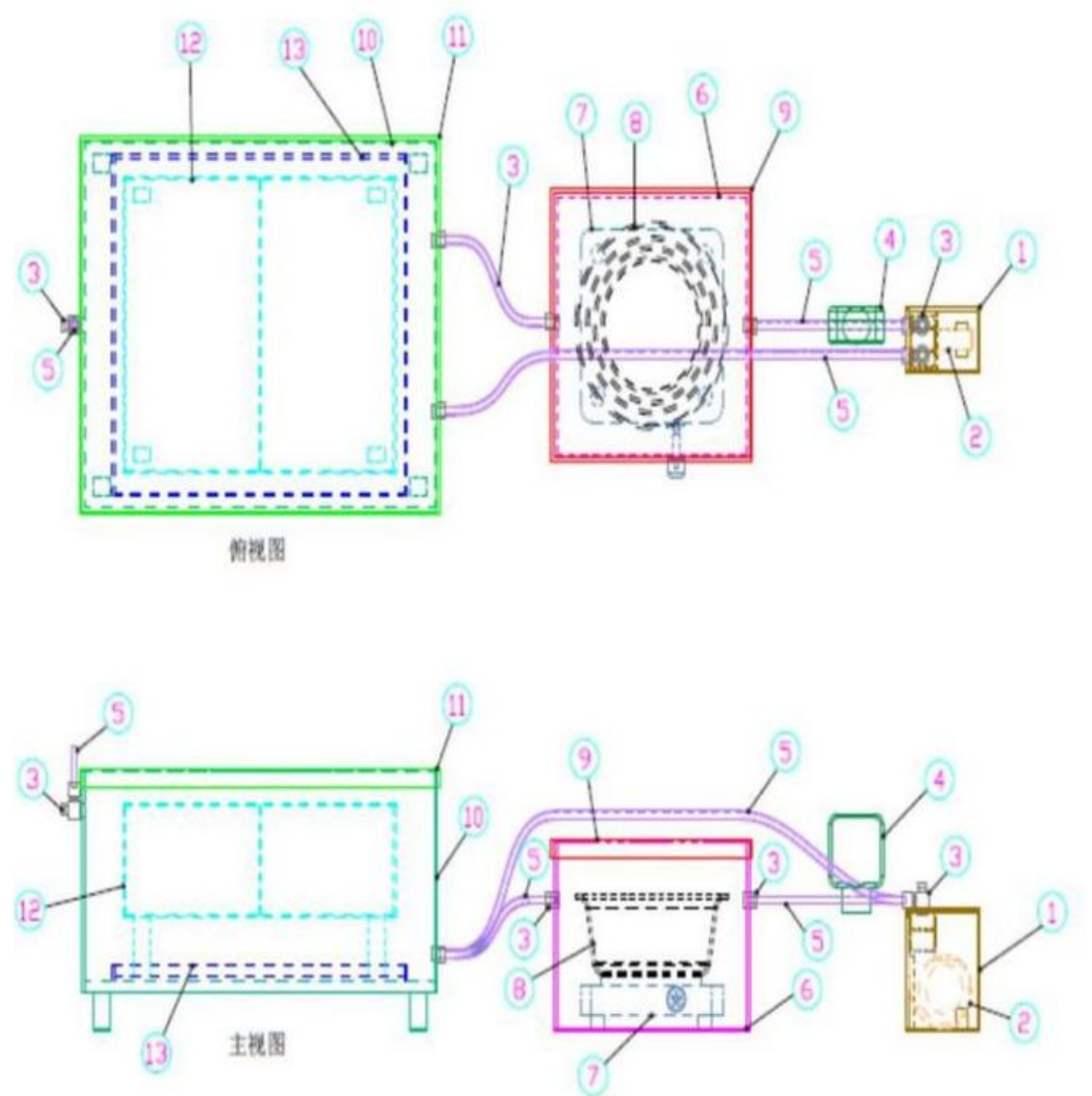

\begin{tabular}{|c|l|c|l|}
\hline 1 & Blast box & 7 & electric oven \\
\hline 2 & blower & 8 & wok \\
\hline 3 & gas nozzle & 9 & oven lid \\
\hline 4 & gas flow meter & 10 & main box \\
\hline 5 & trachea & 11 & main box lid \\
\hline 6 & oven box & 12 & animal cage \\
\hline 13 & \multicolumn{3}{|c|}{ waste box } \\
\hline
\end{tabular}

Figure 1

odor administration instrument 
A1

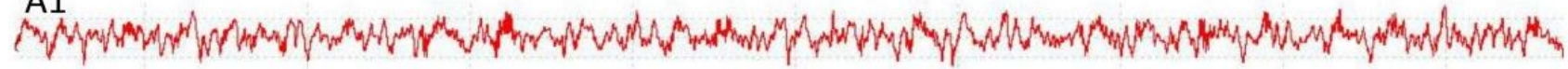

A2

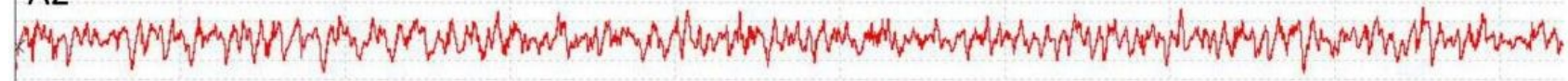

A3

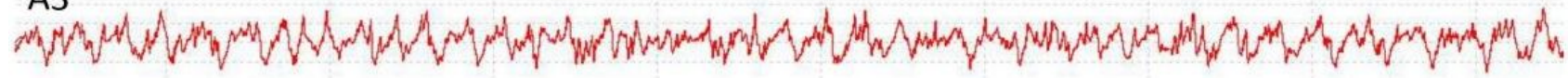

A4

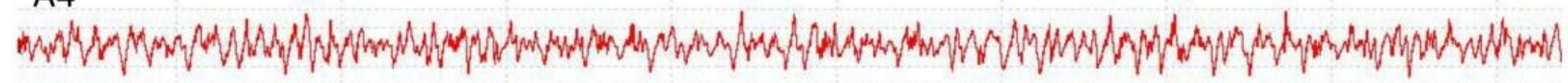

A5

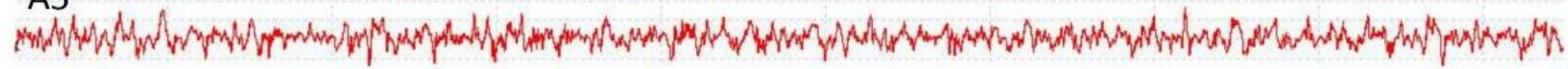

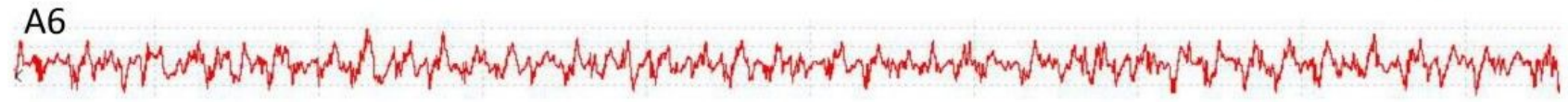

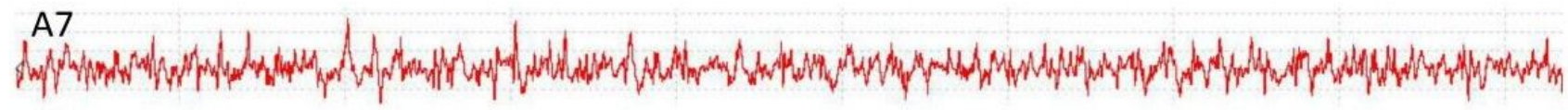

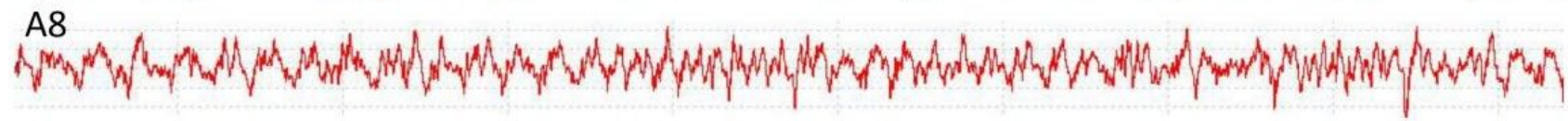

B

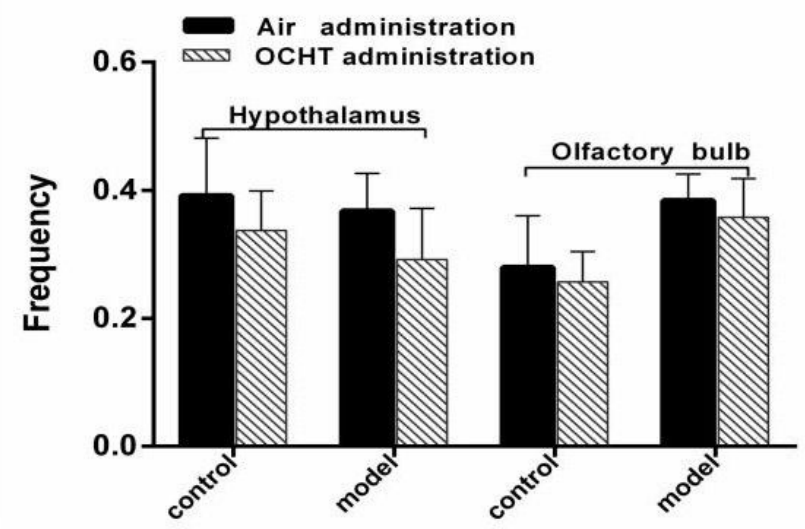

C
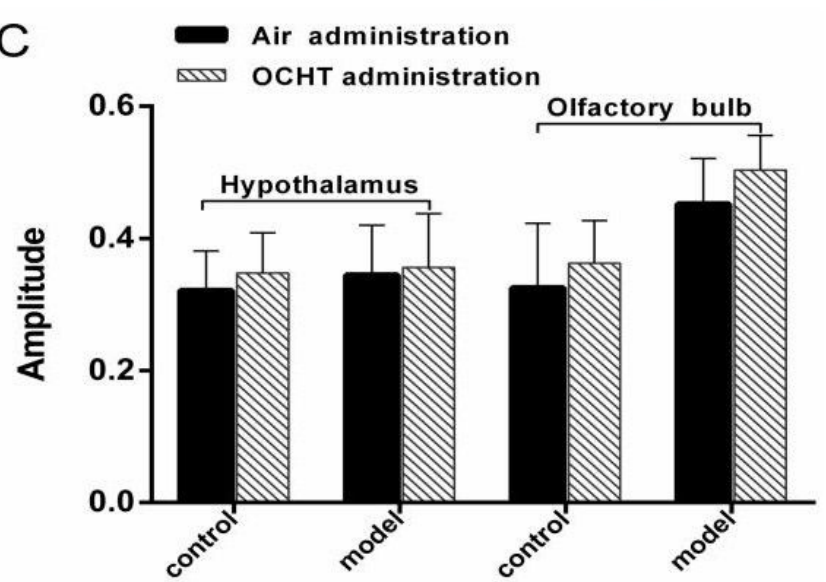

Figure 2

Effects of the odor of charred hawthorn (OCHT) on hypothalamus and olfactory bulb in rats. (A) Electroencephalogram in hypothalamus and olfactory bulb of rats; (A1) Effects of clean air on electroencephalogram in hypothalamus of rats in control group; (A2) Effects of OCHT on electroencephalogram in hypothalamus of rats in control group; (A3) Effects of clean air on electroencephalogram in hypothalamus of rats in model group; (A4) Effects of OCHT on electroencephalogram in hypothalamus of rats in model group; (A5) Effects of clean air on electroencephalogram in olfactory bulb of rats in control group; (A6) Effects of OCHT on electroencephalogram in olfactory bulb of rats in control group; (A7) Effects of clean air on electroencephalogram in olfactory bulb of rats in model group; (A8) Effects of OCHT on electroencephalogram in olfactory bulb of rats in model group. (B) Effects of clean air and OCHT on EEG frequency of hypothalamus and olfactory bulb in rats. (C) Effects of clean air and OCHT on EEG amplitude of hypothalamus and olfactory bulb in rats. 


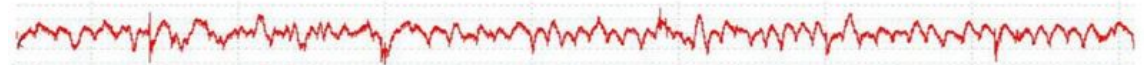

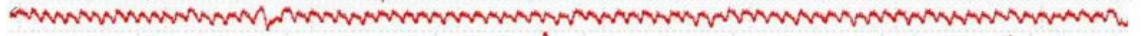

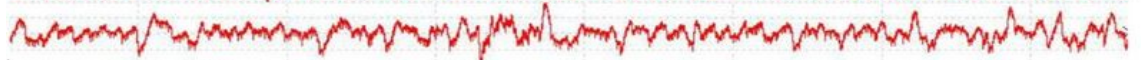

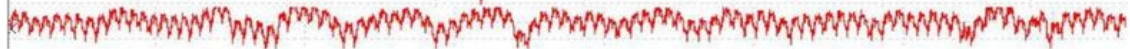

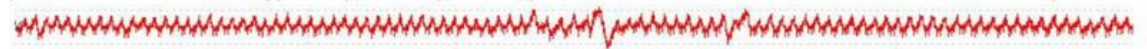
(1)

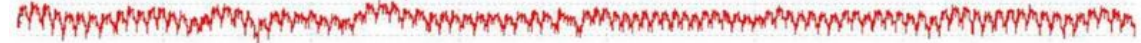

A1

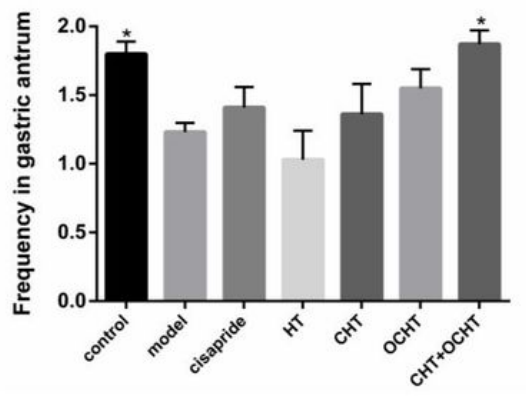

A2

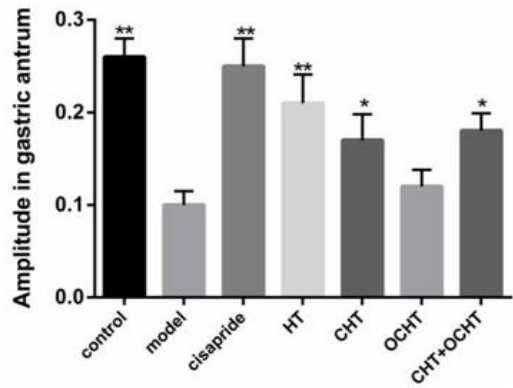

B

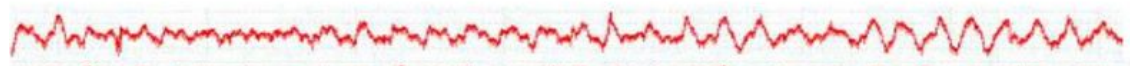

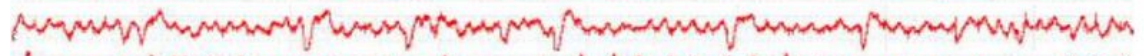

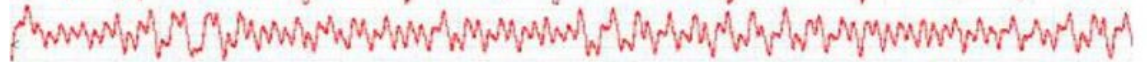

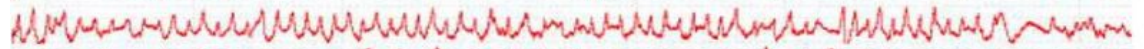

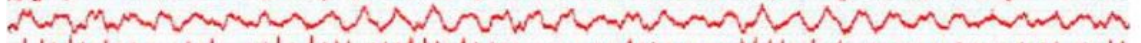

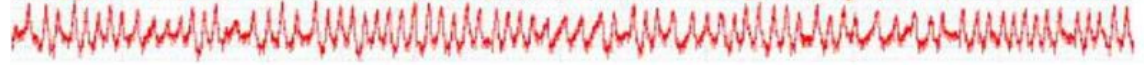
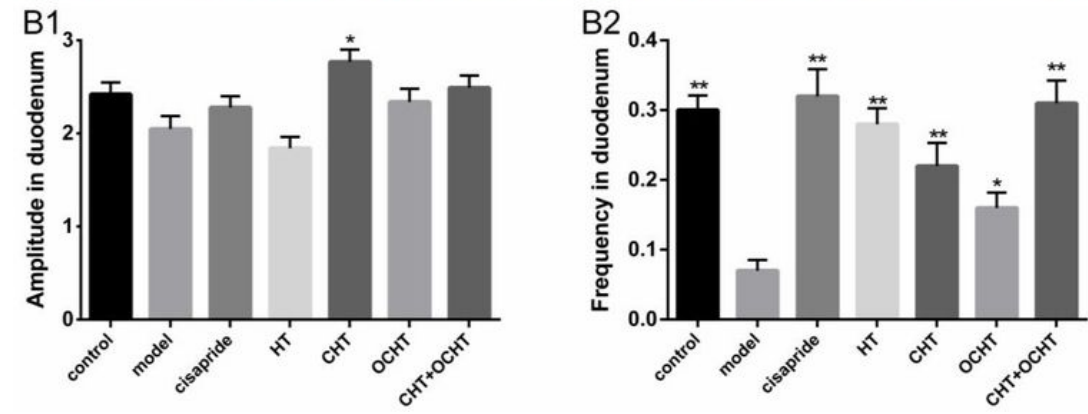

Figure 3

Electrogastrography (EGG) in gastric antrum and duodenal of rats. (A) EGG in gastric antrum of rats, including control group, model group, cisapride group, HT group, $\mathrm{CHT}$ group, OCHT group from top to bottom; (A1) EGG frequency in gastric antrum of rats; (A2) EGG amplitude in gastric antrum of rats. (B) EGG in duodenal of rats, including control group, model group, cisapride group, $\mathrm{HT}$ group, $\mathrm{CHT}$ group, $\mathrm{OCHT}$ group and $\mathrm{CHT}+\mathrm{OCHT}$ group from top to bottom; (B1) EGG frequency in duodenal of rats; (B2) EGG amplitude in duodenal of rats. HT: hawthorn group, CHT: charred hawthorn group, OCHT:odor of charred hawthorn group, $\mathrm{CHT}+\mathrm{OCHT}$ : charred hawthorn + odor of charred hawthorn group. Data were analyzed by one-way ANOVA (mean $\pm S D, n=6$ ).SPSS 17.0was used for statistical analyses. VS model group, ${ }^{\star} P<0.05,{ }^{\star *} P<0.01$. 
Group
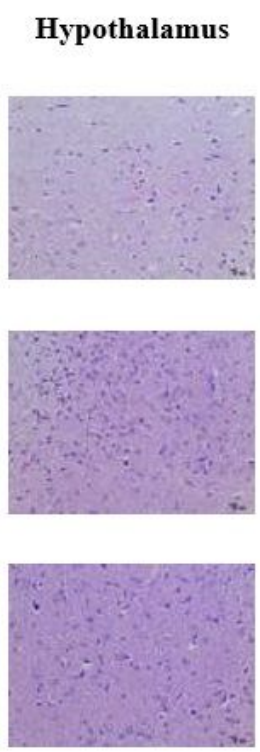

Cisapride

HT
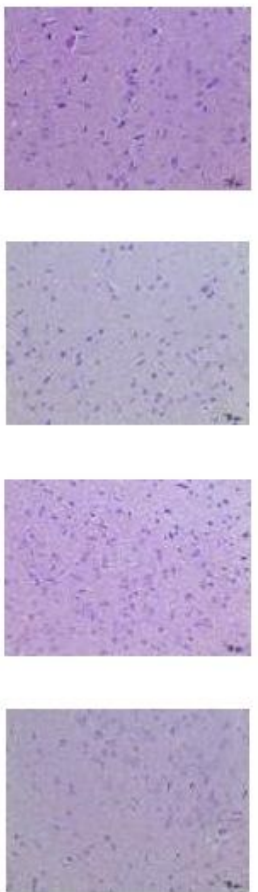
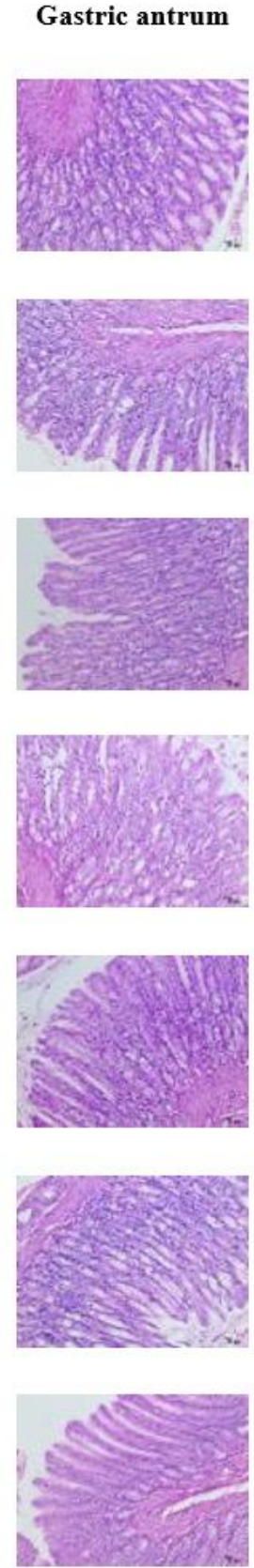

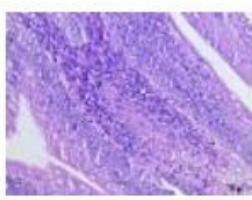

Duodenum
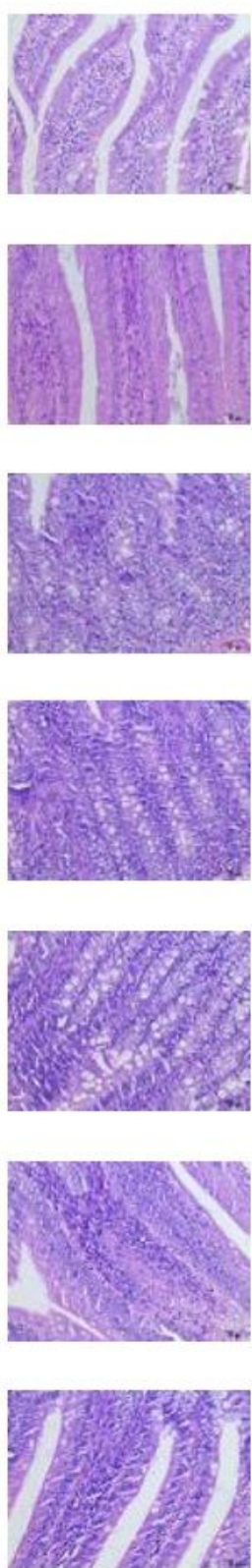

\section{Figure 4}

Histopathological examination of rats in hypothalamus, gastric antrum and duodenum tissue. There were 6 rats in each group, half male and half female. HT: hawthorn group, CHT: charred hawthorn group, OCHT: the odor of charred hawthorn, $\mathrm{CHT}+\mathrm{OCHT}$ : charred hawthorn + odor of charred hawthorn group. 


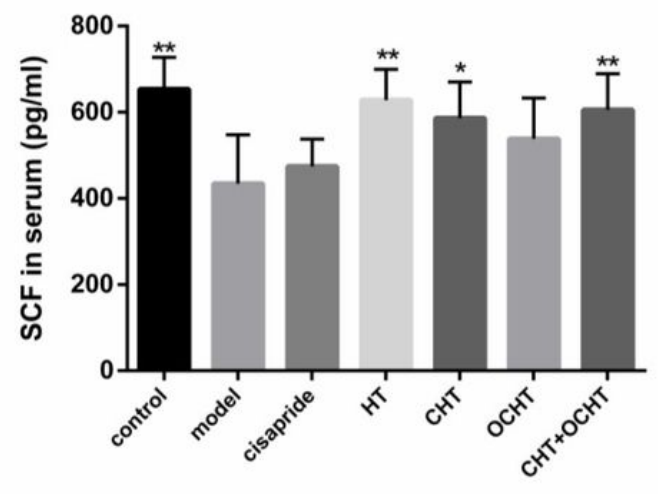

B

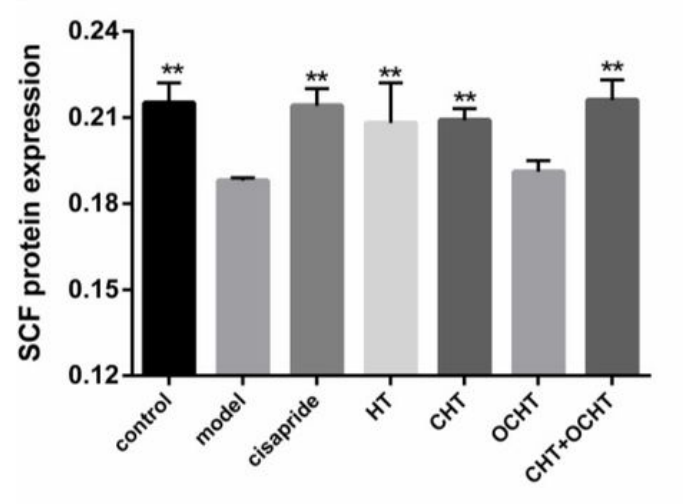

C
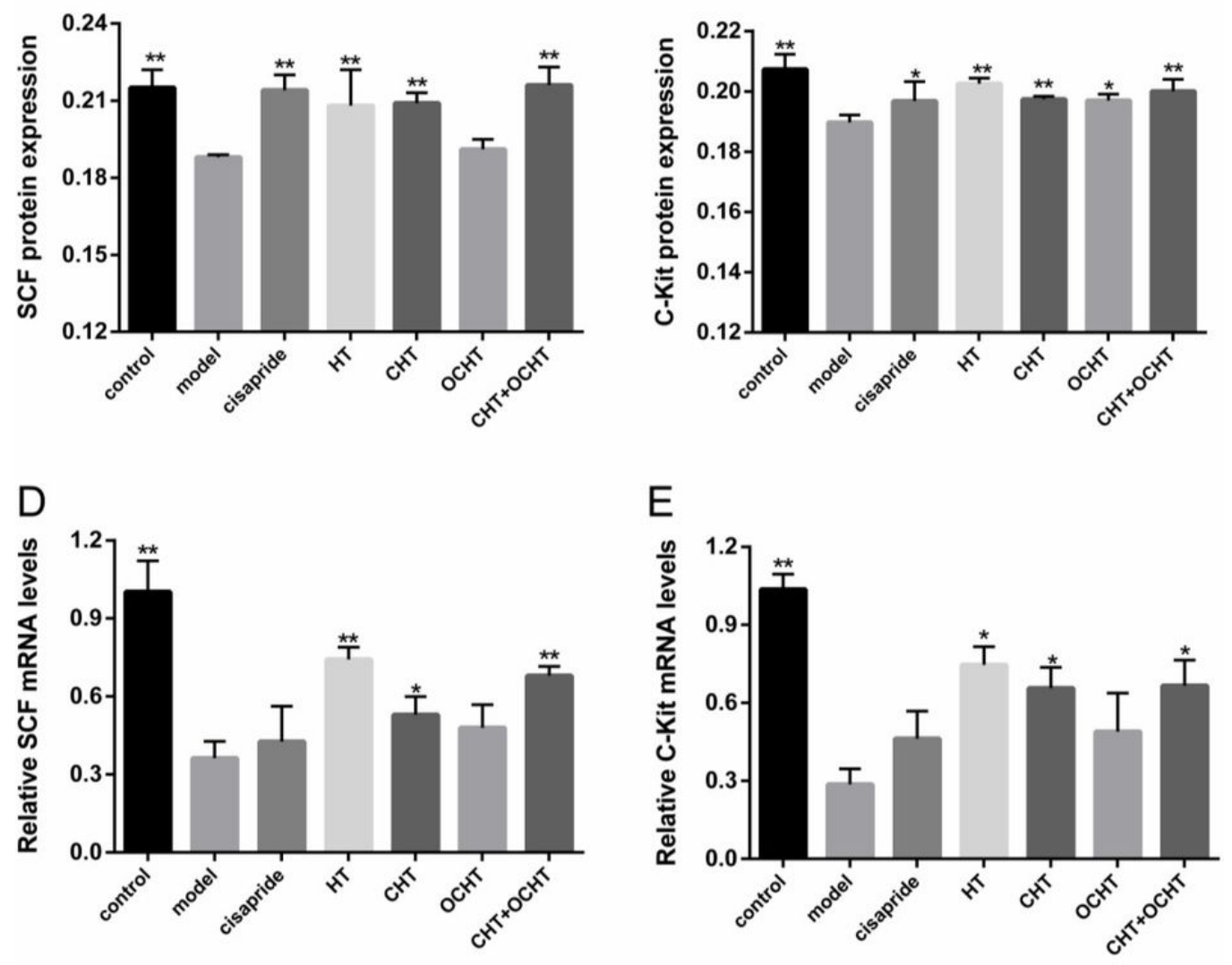

Figure 5

The effect of hawthorn on SCF/C-Kit pathways in rats with HC-DID. (A) Serum SCF in rats. (B) SCF protein expression in gastric antrum of rats. (C) C-Kit protein expression in gastric antrum of rats. (D) Relative SCF mRNA levels in gastric antrum of rats. (E) Relative C-Kit mRNA levels in gastric antrum of rats. HT: hawthorn group, CHT: charred hawthorn group, OCHT: odor of charred hawthorn, $\mathrm{CHT}+\mathrm{OCHT}$ : charred hawthorn + odor of charred hawthorn group. Data were analyzed by one-way ANOVA (mean $\pm S D, n=6$ ). SPSS 17.0was used for statistical analyses. VS model group, ${ }^{\star} P<0.05,{ }^{\star} * P<0.01$.

\section{Supplementary Files}

This is a list of supplementary files associated with this preprint. Click to download.

- SupplementaryMaterial.pdf

- SupplementaryMaterials.docx 